\title{
Laser-Controlled Fluorescence in Two-Level Systems
}

\author{
Jamie M. Leeder, David S. Bradshaw, and David L. Andrews* \\ School of Chemistry, University of East Anglia, Norwich NR4 7TJ, United Kingdom \\ *To whom correspondence should be addressed. E-mail: d.1.andrews@uea.ac.uk
}

\section{RECEIVED DATE}

The ability to modify the character of fluorescent emission by a laser-controlled, optically nonlinear process has recently been shown theoretically feasible, and several possible applications have already been identified. In operation, a pulse of off-resonant probe laser beam, of sufficient intensity, is applied to a system exhibiting fluorescence, during the interval of excited state decay following the initial excitation. The result is a rate of decay that can be controllably modified, the associated changes in fluorescence behavior affording new, chemically-specific information. In this paper, a two-level emission model is employed in the further analysis of this all-optical process; the results should prove especially relevant to the analysis and imaging of physical systems employing fluorescent markers these ranging from quantum dots to green fluorescence protein. Expressions are presented for the lasercontrolled fluorescence anisotropy exhibited by samples in which the fluorophores are randomly oriented. It is also shown that, in systems with suitably configured electronic levels and symmetry properties, fluorescence emission can be produced from energy levels that would normally decay nonradiatively. 


\section{Introduction}

In any molecular system that exhibits fluorescence, the primary result of ultraviolet/visible absorption is the electronic excitation of individual fluorophores. Typically, ultrafast intramolecular vibrational redistribution processes produce a degree of immediate relaxation with a partial degradation of the acquired energy, subsequent fluorescence occurring from the lowest level of the electronic excited state. As is well known, the throughput of a laser beam in such photo-activated systems can produce stimulated emission when the optical frequency matches the fluorescence, a phenomenon that has found analytical applications in stimulated emission depletion spectroscopy. ${ }^{1-7}$ However, it has recently emerged that a moderately intense, completely off-resonant probe laser beam can also significantly affect process of fluorescence. ${ }^{8-11}$ Under such conditions the probe essentially confers optical nonlinearity on the fluorescent emission, and in consequence each excited-state lifetime, $\tau$, is appreciably modified.

The essence of the effect can be captured in a very simple general formula, $\tau^{-1}=\tau_{\mathrm{fl}}^{-1}+\tau_{\mathrm{nr}}^{-1}+\kappa I$; the first two terms on the right correspond to inverses of the excited-state lifetimes for fluorescence and competing non-radiative decay, respectively, whilst the effect of the probe emerges in the form of the additional term proportional to $I$, the irradiance of the off-resonant probe. In a heterogeneous sample the above constant of proportionality, $\kappa$, which is determined by detailed molecular nonlinearity, will generally take a different value for each chemically distinct component. One can draw some analogy with the well-known enhancement of emission, which can occur through coupling with strong electric fields. ${ }^{12-14}$ However, the newly discovered mechanism signifies similar modifications to spontaneous fluorescent emission that are produced without the presence of any surface or static field, through direct interaction with the oscillating electric field of throughput electromagnetic radiation. Initial estimates have suggested that fluorescence lifetimes, under specified conditions, can be reduced by $10 \%$ or more, for an input laser irradiance of $10^{11} \mathrm{~W} \mathrm{~cm}^{-2}$ (with typical values of $16 \times 10^{-30} \mathrm{C} \mathrm{m}$ for the magnitude of 
the transition dipole moment and a photonic energy as $\left.10^{-19} \mathrm{~J}\right){ }^{8}$ so that the effect should be readily amenable to measurement with modulation-based instrumentation. In systems with suitably configured electronic levels and symmetry properties, it is also possible for fluorescence emission to be produced from energy levels that would normally decay non-radiatively.

In this paper we report in detail on how, in systems of randomly oriented fluorophores, the effects of laser-controlled fluorescence will be manifest in changes to the emission anisotropy. Our methods are developed from a fully quantum mechanical analysis of the molecular electrodynamics, a methodology whose value has been brought to the fore and proven in numerous works by Mukamel - see for example refs $15-21$. We begin in section 2 with a recap on the theory of laser-controlled fluorescence. In section 3 , it is shown how a two-level formulation of theory can be implemented using an expedient, entirely rigorous procedural algorithm that highlights the twin dependence on static and transition dipole moments. Using this method, we secure tractable expressions whose broad validity will extend from quantum dots $\operatorname{dos}^{22,23}$ to fluorescent proteins $^{24-28}$ - indeed any material whose emission spectrum is dominated by one excited electronic state. In section 4, following an outline of the procedures for performing the requisite rotational averages, precise expressions are duly presented for the modified fluorescence anisotropy, characterizing and quantifying the probe control mechanism. A brief discussion of the results is given by way of conclusion (section 5).

\section{Laser-controlled fluorescence}

Fluorescence that occurs through spontaneous emission generally involves a single molecule-photon interaction (Fig. 1), and its representation in theory is cast in terms of first-order time-dependent perturbation theory. In circumstances where no other light is present - a condition which, in normal experiments, is satisfied once the radiation responsible for the initial electronic excitation has passed out 




Fig. 1. Energy level representation for spontaneous fluorescence. Electronic states (and their vibrational manifolds) are signified by the boxes, the wavy line is the emitted fluorescence $\left(\hbar \omega^{\prime}\right)$ and the vertical arrow is a transition due to the emission. Energy levels $E_{0}$ and $E_{\alpha}$ denote the ground and excited molecular states, respectively, and the dot symbolizes a single molecule-photon interaction.

of the system - then higher order (odd-rank) perturbation terms are usually insignificant, only denoting self-energy corrections. However, higher-order interactions will also arise on application of an offresonant probe laser, namely where a laser wavelength is chosen at which the fluorophores are optically transparent. There is no net absorption or stimulated emission of such a beam, but elastic forwardscattering events do occur - photons are annihilated and created into the same radiation mode (which thus emerges unchanged). Such events can engage by nonlinear coupling with the fluorescence emission, resulting in three concerted molecule-photon interactions (Fig. 2), i.e. an overall process whose quantum amplitude is determined by third-order perturbation theory. Similar effects occur in connection with resonance energy transfer, as numerous studies have shown; ${ }^{29-38}$ such behavior is 


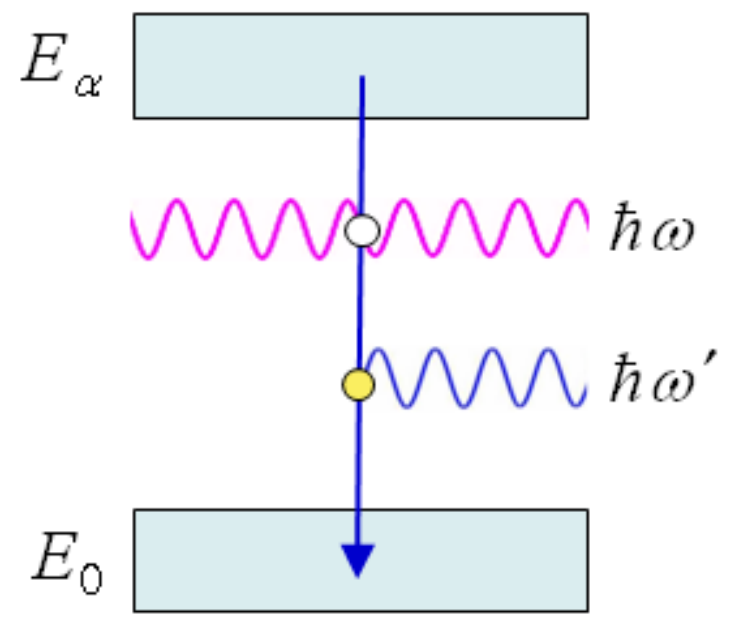

Fig. 2. Energy level representation for the nonlinear coupling mechanism. As Fig. 1, but also including the off-resonant laser beam $(\hbar \omega)$ denoted by the upper wavy line; the upper dot symbolizes two concerted molecule-photon interactions (i.e. elastic forward-scattering).

nonetheless in contrast to the perturbations that can be brought into effect by a static field, the leading orders of which arise in second-order, i.e. linear in both the emitted and the applied field.

The intensity of fluorescence, $I^{\prime}\left(\Omega^{\prime}\right)$, (or power per unit solid angle) follows from the Fermi Rule, ${ }^{39}$ the associated rate being multiplied by the energy of a fluorescence photon, $\hbar \omega^{\prime} \equiv \hbar c k^{\prime} .{ }^{40,41}$ The result signifies the signal that is produced by a single molecule initially in the relevant excited state. By inclusion of the mechanism under present scrutiny, the net intensity is hence determined from $I^{\prime}\left(\Omega^{\prime}\right) \mathrm{d} \Omega^{\prime}=2 \pi \rho c k^{\prime}\left|M_{\mathrm{fl}}^{(1)}+M_{\mathrm{fl}}^{(3)}\right|^{2}$, where $M_{\mathrm{fl}}^{(1)}$ and $M_{\mathrm{fl}}^{(3)}$ are the quantum amplitudes for first- and third-order interaction processes, respectively, and the density of radiation states is 
$\rho=\left(k^{\prime 2} V / 8 \pi^{3} \hbar c\right) \mathrm{d} \Omega^{\prime}$. As determined elsewhere, ${ }^{9}$ from this expression a general representation for the intensity of laser-controlled fluorescence is found, and is given by;

$$
\begin{aligned}
I^{\prime}\left(\Omega^{\prime}\right) & =\left(\frac{c k^{\prime 4}}{8 \pi^{2} \varepsilon_{0}}\right)\left[e_{i}^{\prime} e_{j}^{\prime} \mu_{i}^{0 \alpha} \bar{\mu}_{j}^{0 \alpha}+\left(I / c \varepsilon_{0}\right) e_{i} e_{j} e_{k}^{\prime} e_{l}^{\prime} \chi_{i j k}^{0 \alpha}\left(\omega^{\prime} ;-\omega, \omega\right) \bar{\mu}_{l}^{0 \alpha}\right. \\
& \left.+\left(I^{2} / 4 c^{2} \varepsilon_{0}^{2}\right) e_{i} e_{j} e_{k}^{\prime} e_{l} e_{m} e_{n}^{\prime} \chi_{i j k}^{0 \alpha}\left(\omega^{\prime} ;-\omega, \omega\right) \bar{\chi}_{l m n}^{0 \alpha}\left(\omega^{\prime} ;-\omega, \omega\right)\right],
\end{aligned}
$$

where the fluorescence-decay transition dipole moment is designated by the shorthand notation $\boldsymbol{\mu}^{0 \alpha}=\langle 0|\boldsymbol{\mu}| \alpha\rangle-$ in which $|\alpha\rangle$ denotes the excited state, and $|0\rangle$ the ground state. The nonlinear transition susceptibility $\chi_{i j k}^{0 \alpha}\left(\omega^{\prime} ;-\omega, \omega\right)$ is defined below. In equation (1), the implied summation convention for repeated Cartesian tensor (subscript) indices is used, and $I$ is the irradiance of the laser probe, with $\mathbf{e}^{\prime}$ and $\mathbf{e}$ representing the polarization vectors of fluorescence and probe photons, respectively. For simplicity, all photons are assumed linearly polarized.

The initial term on the right-hand side of Eq. (1) corresponds to spontaneous emission, intrinsic to the system and independent of the probe laser beam; the last term signifies a coupling of the elastically forward-scattered probe beam with the fluorescence emission. The middle term, linear in $I$, signifies a quantum interference of these two concurrent processes; the overall multiplier of $I$ in this term can be identified with $\hbar c k^{\prime}$ times the $\kappa$ that appeared in the equation for excited state lifetime, in Section 1 . In principle, measuring the effect of the passive beam at varying levels of intensity should enable the value of $\kappa$ to be experimentally determined. In general, it may be assumed that the leading term in (1) is non-zero and the middle one is the leading correction, although a configuration is possible in which the third term exists on its own, i.e. when the first and second terms are null; this will be discussed in detail in section 4.3 . 
Continuing, the sum-over-states form of the third-order nonlinear optical 'transition hyperpolarizability' tensor, explicitly exhibiting the frequency dispersion, is as follows;

$$
\begin{aligned}
\chi_{i j k}^{0 \alpha}\left(\omega^{\prime} ;-\omega, \omega\right)= & \sum_{r} \sum_{s \neq \alpha}\left(\frac{\mu_{k}^{0 s} \mu_{j}^{s r} \mu_{i}^{r \alpha}}{\tilde{E}_{s \alpha}\left(\tilde{E}_{r \alpha}-\hbar \omega\right)}+\frac{\mu_{k}^{0 s} \mu_{i}^{s r} \mu_{j}^{r \alpha}}{\tilde{E}_{s \alpha}\left(\tilde{E}_{r \alpha}+\hbar \omega\right)}\right) \\
& +\sum_{r} \sum_{s}\left(\frac{\mu_{j}^{0 s} \mu_{k}^{s r} \mu_{i}^{r \alpha}}{\left(\tilde{E}_{s \alpha}-\hbar \omega+\hbar \omega^{\prime}\right)\left(\tilde{E}_{r \alpha}-\hbar \omega\right)}+\frac{\mu_{i}^{0 s} \mu_{k}^{s r} \mu_{j}^{r \alpha}}{\left(\tilde{E}_{s \alpha}+\hbar \omega+\hbar \omega^{\prime}\right)\left(\tilde{E}_{r \alpha}+\hbar \omega\right)}\right) \\
& +\sum_{r \neq 0} \sum_{s}\left(\frac{\mu_{j}^{0 s} \mu_{i}^{s r} \mu_{k}^{r \alpha}}{\left(\tilde{E}_{s \alpha}-\hbar \omega+\hbar \omega^{\prime}\right)\left(\tilde{E}_{r \alpha}+\hbar \omega^{\prime}\right)}+\frac{\mu_{i}^{0 s} \mu_{j}^{s r} \mu_{k}^{r \alpha}}{\left(\tilde{E}_{s \alpha}+\hbar \omega+\hbar \omega^{\prime}\right)\left(\tilde{E}_{r \alpha}+\hbar \omega^{\prime}\right)}\right),
\end{aligned}
$$

where $\omega$ is the probe beam frequency, $r$ and $s$ are intermediate molecular states, $E_{x y}=E_{x}-E_{y}$ is an energy difference between two such states (for example $E_{\alpha 0} \equiv \hbar \omega^{\prime} \equiv \hbar c k^{\prime}$ ) and the transition moments are defined in the same manner as $\mu^{0 \alpha}$. The tildes serve as a reminder to add to the excited state energies, in the case of near-resonance conditions, imaginary terms to accommodate line-shape and damping. With reference to later comments, it is worth noting here that there is no assumption of Kleinman symmetry ${ }^{42}$ at this stage - this being a simplifying device, commonly made for calculational expediency, that would impose complete index symmetry for such a tensor.

\section{Two-level systems}

Considering the dependence of the fluorescence signal on the optical frequency of the probe, it is evident that the denominators within the transition hyperpolarizability tensor of equation (2) are primarily responsible for determining any degree of enhancement or suppression of the fluorescence emission. These factors are ultimately determined by the relative positioning of the fluorophore energy levels, relative to the magnitude of the probe photon energy. To discover more, it is convenient to 
assume that the probe light is delivered in the form of a tunable beam with optical frequency $\omega<\omega^{\prime}$, a condition that specifically precludes single-photon excitation of ground-state molecules. It will also be assumed that the chosen range of probe frequencies cannot produce multiphoton excitation. The main challenge in evaluating the nonlinear response characterized by the transition tensors within equation (1) now lies with implementing the required sum over intermediate states. There is a potentially infinite number of energy levels associated with $r$ and $s$, and to ease calculational complexity it is common to reduce such sets to a small, finite number by approximation. In the present context, it is defensible to consider only the states through which the majority of the optical transitions occur, which in the case of many fluorescent systems limits the selection to just the ground and lowest energy excited states, i.e. a two-state model may be applied. ${ }^{15,16,18,20,22-28,43-52}$ To be clear, the assumption is that the character of the fluorescence emission process, including the effect of the probe radiation, is dominated by two electronic levels; it is not to be presumed that the state from which the fluorescence decay occurs is necessarily the same as the state initially populated by photoexcitation.

Restricting both intermediate states featured within equation (1) to just $|0\rangle$ and $|\alpha\rangle$, only four unique routes can describe virtual transition sequences from the excited to ground molecular states progressing through both $r$ and $s:$ the $\alpha \rightarrow r \rightarrow s \rightarrow 0$ sequences specifically expressible as $\alpha 000, \alpha \alpha 00, \alpha 0 \alpha 0$ and $\alpha \alpha \alpha 0$. Each sequence generates a combination of $0 \leftrightarrow \alpha$ transition electric dipole moments, $\boldsymbol{\mu}^{0 \alpha}$ and $\boldsymbol{\mu}^{\alpha 0}$, in combination with the static dipole moments of the ground and excited energy levels, $\boldsymbol{\mu}^{00}$ and $\boldsymbol{\mu}^{\alpha \alpha}$ respectively. It can be assumed that the former transition electric moments $\boldsymbol{\mu}^{0 \alpha}$ and $\boldsymbol{\mu}^{\alpha 0}$ are real (as is always possible, given a suitable choice of basis set for the molecular wavefunctions - for the present we exclude considerations of electronic degeneracy) and therefore equal, by virtue of the Hermiticity of the dipole operator. Detailed analysis reveals that the dependence on permanent moments emerges only in terms of their vector difference, $\mathbf{d}=\boldsymbol{\mu}^{\alpha \alpha}-\boldsymbol{\mu}^{00}$, i.e. the shift in static dipole moment that accompanies the transition. (Indeed, this feature applies to all nonlinear optical 
susceptibilities, treated by a two-level model.) With the benefit of an algorithmic method, the following prescription can be adopted: ${ }^{53-55}$

$$
\boldsymbol{\mu}^{\alpha \alpha} \rightarrow \boldsymbol{\mu}^{\alpha \alpha}-\boldsymbol{\mu}^{00}=\mathbf{d} ; \boldsymbol{\mu}^{00} \rightarrow 0,
$$

whose general validity has been proven to rest on a canonical transformation of the quantum interaction operator. ${ }^{56}$ Applying this protocol requires application of an associated rule: any transitional mechanism that connects the initial and final system states (here, for the emission process) through a ground state static dipole is to be discarded, and hence only two of the originally proposed four sequences, namely $\alpha 0 \alpha 0$ and $\alpha \alpha \alpha 0$ persist. Applied to the six terms within equation (2), the two-level hyperpolarizability tensor is generally expressible as a sum of 12 separate contributions. Further simplification ensues because a number of these terms, when $r=0$ and/or $s=\alpha$, are precluded by the conditions of perturbation theory, namely the exclusion of virtual states that equate to the initial or final state. The two-state form of $\chi_{i j k}^{0 \alpha}\left(\omega^{\prime} ;-\omega, \omega\right)$ thus re-emerges as:

$$
\chi_{i j k}^{0 \alpha}\left(\omega^{\prime} ;-\omega, \omega\right)=\frac{2}{\hbar^{2}} \frac{\mu_{j}^{0 \alpha} \mu_{k}^{0 \alpha} \mu_{i}^{0 \alpha}}{\left(\omega^{2}-\omega^{\prime 2}\right)}+\frac{\mu_{j}^{0 \alpha} d_{i} d_{k}}{\hbar^{2} \omega \omega^{\prime}}-\frac{\mu_{i}^{0 \alpha} d_{j} d_{k}}{\hbar^{2} \omega \omega^{\prime}}
$$

It may be observed that the second and third terms on the right in equation (4) exhibit an antisymmetry with respect to interchange of the indices $i$ and $j$. However, in the physical observable delivered by (1), this tensor is index-contracted with a $i, j$-symmetric product of polarization vectors. Consequently, since only the $i, j$-symmetric part of (4) can contribute to the fluorescence signal, it is expedient to replace $\chi_{i j k}^{0 \alpha}\left(\omega^{\prime} ;-\omega, \omega\right)$, without further approximation, by an index-symmetrised form, $\chi_{(i j) k}^{0 \alpha}\left(\omega^{\prime} ;-\omega, \omega\right)$ that is defined as follows: 


$$
\chi_{(i j) k}^{0 \alpha}\left(\omega^{\prime} ;-\omega, \omega\right) \equiv \frac{1}{2}\left(\chi_{i j k}^{0 \alpha}\left(\omega^{\prime} ;-\omega, \omega\right)+\chi_{j i k}^{0 \alpha}\left(\omega^{\prime} ;-\omega, \omega\right)\right)=\frac{2}{\hbar^{2}} \frac{\mu_{i}^{0 \alpha} \mu_{j}^{0 \alpha} \mu_{k}^{0 \alpha}}{\left(\omega^{2}-\omega^{\prime 2}\right)}
$$

It is notable that the expression on the right is, in fact, fully index-symmetric, i.e. symmetric with respect to interchange of any pair of indices. We therefore observe that the two-level model delivers a result that is consistent with the adoption of Kleinman symmetry, even though the latter condition has not been artificially imposed. Furthermore, there is a significant physical consequence; it emerges that the physical mechanism for the laser-controlled emission depends only on the transition dipole, and not on the static moments. In passing we note that a low-frequency, $\omega \rightarrow 0$, limit of the above analysis requires caution, because in this limit some of the intermediate system states, allowed for a finite $\omega$, become identifiable with the initial or final state of the process, and are necessarily removed from the sum over states. However, the ensuing result is of little interest since it represents only a correction to the more prominent response - which arises in second order perturbation theory, as noted earlier.

\section{Fluorescence anisotropy}

We now turn our attention to optical polarization features. As is well established, there is a great deal of important information, highly relevant to speciation and structure determination, which can be derived from fluorescence anisotropy. Specifically, the anisotropy parameters signify the degree to which fluorescence retains a directionality of polarization from the initial excitation - see for example chapter 5 of the classic text by Valeur. ${ }^{57}$ The associated experimental measurements can also inform on excited state photophysical processes such as internal conversion, hindered rotation, rotational diffusion, intramolecular energy transfer etc. Each of these processes represents one of the means by which the character of fluorescent emission can differ from that of the preceding absorption - quite apart from the Stokes shift in wavelength that is normally apparent. The former processes all provide situations in 
which the emission dipole moment need not be parallel to the absorption moment. To accommodate such features in the present theory, the initial absorption must now be incorporated into our analysis. Since the probe beam is only delivered to the system after the initial excitation, we have:

$$
\left\langle I^{\prime}\left(\Omega^{\prime}\right)\right\rangle \sim\left\langle\left|M_{\mathrm{abs}}^{(1)}\right|^{2}\left|M_{\mathrm{fl}}^{(1)}+M_{\mathrm{fl}}^{(3)}\right|^{2}\right\rangle
$$

Here, the subscript abs denotes the single-photon absorption mechanism, and the angular brackets denote an orientational average to account for the fact that the molecular transition moments associated with absorption and emission (the latter duly modified by the probe), although correlated within the molecular frame, are together randomly oriented relative to the input propagation. The structure of equation (6) provides for the excitation and emission processes to be separable in time. In more detail, the quantum amplitude $M_{\text {abs }}^{(1)}$ corresponding to the initial absorption is proportional to $\mathbf{e}_{0} \bullet \boldsymbol{\mu}^{\nu 0}$, where $\mathbf{e}_{0}$ represents the input polarization vector aligned in the $z$-direction by definition, and $|v\rangle$ designates the state initially populated by the excitation. As indicated above, the latter may or may not be the same as the electronic state from which subsequently emission occurs, depending on factors such as the possibility of intervening relaxation or intramolecular energy transfer.

The anisotropy is now determined from the general expression $r^{\prime}=\left(\left\langle I_{\|}^{\prime}\right\rangle-\left\langle I_{\perp}^{\prime}\right\rangle\right) /\left(\left\langle I_{\|}^{\prime}\right\rangle+2\left\langle I_{\perp}^{\prime}\right\rangle\right)$, where $\left\langle I_{\|}^{\prime}\right\rangle$ and $\left\langle I_{\perp}^{\prime}\right\rangle$ are the components of fluorescence intensity polarized parallel and perpendicular, respectively, to the electric vector of the initial excitation beam. ${ }^{57}$ In the present context, this requires detailed examination of the tensor contractions within equation (1), and the performance of orientational averages. 


\subsection{First order correction}

In equation (1), we recall that the first term represents single-photon induced fluorescence, the last term corresponding to the process modified by the off-resonant laser throughput; the second term signifies a quantum interference of these two processes, the lead correction. For present purposes it is assumed that the third term under these conditions represents a comparatively small contribution; its contribution is considered later. The most computationally effective procedure for implementing the necessary orientational averages is well established - see for example Appendix 2 of ref. 41 . It involves intricate matrix algebra whose scale escalates rapidly with rank. Thus, whereas the leading term associated with regular fluorescence requires only a fourth-rank tensor average (cast in terms of isotropic tensors as a $3 \times$ 3 matrix) the correction requires a sixth-rank average $(15 \times 15)$. On completion, the following result emerges:

$$
\begin{aligned}
\left\langle I^{\prime}\left(\Omega^{\prime}\right)\right\rangle= & \Xi\left(I_{0}, \omega_{0}\right)\left[T_{i i} \bar{T}_{j j}\left(3 \cos ^{2} \phi-1\right)+T_{i j} \bar{T}_{i j}\left(-\cos ^{2} \phi+2\right)\right. \\
& +\frac{I}{7 c \varepsilon_{0}}\left(2 T_{i(i j) j} \bar{T}_{k k}\left(9 \cos \theta \cos \varphi \cos \phi-3 \cos ^{2} \theta-3 \cos ^{2} \varphi-3 \cos ^{2} \phi+2\right)\right. \\
& +T_{i(i j) k} \bar{T}_{j k}\left(-12 \cos \theta \cos \varphi \cos \phi+11 \cos ^{2} \theta+4 \cos ^{2} \varphi+4 \cos ^{2} \phi-5\right) \\
& +2 T_{i(i j) k} \bar{T}_{k j}\left(9 \cos \theta \cos \varphi \cos \phi-3 \cos ^{2} \theta-3 \cos ^{2} \varphi-3 \cos ^{2} \phi+2\right) \\
& +T_{i(j j) i} \bar{T}_{k k}\left(-12 \cos \theta \cos \varphi \cos \phi+4 \cos ^{2} \theta+4 \cos ^{2} \varphi+11 \cos ^{2} \phi-5\right) \\
& +T_{i(j j) k} \bar{T}_{i k}\left(8 \cos \theta \cos \varphi \cos \phi-5 \cos ^{2} \theta-5 \cos ^{2} \varphi-5 \cos ^{2} \phi+8\right) \\
& \left.\left.+T_{i(j k) j} \bar{T}_{i k}\left(-12 \cos \theta \cos \varphi \cos \phi+4 \cos ^{2} \theta+11 \cos ^{2} \varphi+4 \cos ^{2} \phi-5\right)\right)\right],
\end{aligned}
$$

where $\Xi\left(I_{0}, \omega_{0}\right)$ is a constant of proportionality whose value is determined by the initial (excitation) beam irradiance $I_{0}$ and its corresponding optical frequency $\omega_{0}$; overbars denote complex conjugation. In the above expression, the first two terms signify the usual response, subsequent terms representing the leading corrections produced by the probe. The equation is explicitly cast in terms of the three distinct 
angles between each pair of polarization vectors, for the incident, off-resonant probe and emitted light: $\theta=\cos ^{-1}\left(\mathbf{e}_{0} \cdot \mathbf{e}\right), \varphi=\cos ^{-1}\left(\mathbf{e} \cdot \mathbf{e}^{\prime}\right)$ and $\phi=\cos ^{-1}\left(\mathbf{e}_{0} \cdot \mathbf{e}^{\prime}\right)$. The result also utilizes a shorthand notation to describe the products of molecular transition moments, where for example $T_{i j}$ represents $\mu_{i}^{\nu 0} \mu_{j}^{0 \alpha}$, whilst $T_{i(i j) j}$ corresponds to $\mu_{i}^{\nu 0} \chi_{(i j) j}^{0 \alpha}$. In these instances and in all subsequent application of this notation, the first index of each $\mathbf{T}$ tensor is associated with the initial molecular excitation.

As shown in the previous Section, taking the two-level form of the nonlinear response tensors has the effect of introducing Kleinman symmetry in each of the optically nonlinear response tensor contributions - based on the symmetric transition hyperpolarizability portrayed in equation (5). It emerges that the six nonlinear response tensor products that feature in equation (7) are no longer linearly independent, and the result can be recast in a simpler form involving just three such products;

$$
\begin{aligned}
\left\langle I^{\prime}\left(\Omega^{\prime}\right)\right\rangle= & \Xi\left(I_{0}, \omega_{0}\right)\left[T_{i i} \bar{T}_{j j}\left(3 \cos ^{2} \phi-1\right)+T_{i j} \bar{T}_{i j}\left(-\cos ^{2} \phi+2\right)\right. \\
& +\frac{I}{7 c \varepsilon_{0}}\left(2 T_{i(i j j)} \bar{T}_{k k}\left(6 \cos \theta \cos \varphi \cos \phi-2 \cos ^{2} \theta-2 \cos ^{2} \varphi+5 \cos ^{2} \phi-1\right)\right. \\
& +2 T_{i(i j k)} \bar{T}_{j k}\left(6 \cos \theta \cos \varphi \cos \phi+5 \cos ^{2} \theta-2 \cos ^{2} \varphi-2 \cos ^{2} \phi-1\right) \\
& \left.\left.+2 T_{i(j i j k)} \bar{T}_{i k}\left(-4 \cos \theta \cos \varphi \cos \phi-\cos ^{2} \theta+6 \cos ^{2} \varphi-\cos ^{2} \phi+3\right)\right)\right],
\end{aligned}
$$

where the following have been applied, $T_{i(i j) j} \bar{T}_{k k}+T_{i(j j)} \bar{T}_{k k}=2 T_{i(i j j)} \bar{T}_{k k}, T_{i(i j) k} \bar{T}_{j k}+T_{i(i j) k} \bar{T}_{k j}=2 T_{i(i j k)} \bar{T}_{j k}$ and $T_{i(j j) k} \bar{T}_{i k}+T_{i(j k) j} \bar{T}_{i k}=2 T_{i(j j k)} \bar{T}_{i k}$. In deriving specific results for independent polarization components, further simplification can now be achieved by writing each of the above molecular tensors explicitly in terms of components of the two transition dipole moments, the photo-selected $\boldsymbol{\mu}^{\nu 0}$ and the emission $\boldsymbol{\mu}^{0 \alpha}$. In the following, we introduce $\beta$ as the angle between these two moments. Assuming that the initial excitation has plane polarization $\mathbf{e}$ in the $x$-direction, the fluorescence is now resolved for 
polarizations $\mathbf{e}^{\prime}$ in the $z$ - and $x$-directions, respectively; the results are as follows. For $\phi=0, \varphi=\pi / 2, \theta=\pi / 2$

$$
\left\langle I_{\|}^{\prime}\left(\Omega^{\prime}\right)\right\rangle=\Xi\left(I_{0}, \omega_{0}\right)\left|\mu^{0 \alpha}\right|^{2}\left|\mu^{v 0}\right|^{2}\left[2 \cos ^{2} \beta+1+\frac{2 I\left|\mu^{0 \alpha}\right|^{2}\left(\cos ^{2} \beta+2\right)}{7 \varepsilon_{0} \hbar^{2} c\left(\omega^{2}-\omega^{\prime 2}\right)}\right],
$$

and for $\phi=\pi / 2, \varphi=0, \theta=\pi / 2$;

$$
\left\langle I_{\perp}^{\prime}\left(\Omega^{\prime}\right)\right\rangle=\Xi\left(I_{0}, \omega_{0}\right)\left|\mu^{0 \alpha}\right|^{2}\left|\mu^{\nu 0}\right|^{2}\left[2-\cos ^{2} \beta+\frac{6 I\left|\mu^{0 \alpha}\right|^{2}\left(3-2 \cos ^{2} \beta\right)}{7 \varepsilon_{0} \hbar^{2} c\left(\omega^{2}-\omega^{\prime 2}\right)}\right] .
$$

Hence, upon substitution of equations (9) and (10) into the general anisotropy expression, we find:

$$
r^{\prime}=\frac{3 \cos ^{2} \beta-1+K I\left|\mu^{0 \alpha}\right|^{2}\left(\cos ^{2} \beta-1\right)}{5+K I\left|\mu^{0 \alpha}\right|^{2}\left(20-11 \cos ^{2} \beta\right) / 7}
$$

where $K=2\left(\varepsilon_{0} \hbar^{2} c\left(\omega^{2}-\omega^{\prime 2}\right)\right)^{-1}$. The above equations (9), (10) and (11) are consistent with results recently reported as equations (5), (6) and (7) of reference 8 - subject to a correction of the latter for omission of the $\left|\mu^{0 \alpha}\right|^{2}$ factors featured in the equations presented here. The discussion and conclusions of that earlier study remain valid and are not revisited here. In the limiting case $I=0$, the well-known expression $^{57} r=(1 / 5)\left(3 \cos ^{2} \beta-1\right)$ is recovered. Generally, however, a change in fluorescence anisotropy can be seen to result from the interaction with the probe beam - although it is to be reemphasized that the state of the latter beam is unaffected. 


\subsection{Inclusion of higher corrections}

Up to this point, the third term in equation (1), quadratically dependent on probe laser intensity, has not been considered in detail as its contribution to the overall fluorescence intensity is generally expected to be negligible. Nevertheless, there can be circumstances in which the third term alone provides the fluorescence response, i.e. when the first and second contributions are null. As we shall see, to address this case requires us to move beyond the two-level approximation. Consider, for example, a system where, following optical excitation, population is efficiently transferred to a state $|\alpha\rangle$ that might normally decay non-radiatively, transitions from $|\alpha\rangle$ to $|0\rangle$ being weak or entirely precluded - for example as a result of inherent geometric or symmetry constraints. Then, terms in equation (1) that feature $\boldsymbol{\mu}^{0 \alpha}$ will not contribute to the observed emission, which instead is activated solely in response to the off-resonant throughput. Clearly, the two-level model would also predict a vanishing response from the probe laser, due to the associated structure of the transition hyperpolarizability (5). However the more general analysis, accommodating higher energy levels in the sum over states, allows the possibility of a decay transition that is symmetry allowed by three-photon selection rules.

An outline for an all-optical switch based on laser-controlled fluorescence may be described as follows: (i) an individual molecule is indirectly excited to a 'dark' state (i.e. one whose direct dipolar excitation from the ground state is forbidden); (ii) precluded by the one-photon dipole selection rules, fluorescence occurs from this 'dark' state through optical nonlinear activation only; (iii) this activation arises due to the presence of the intense non-resonant laser field, i.e. the relevant molecular transitions are assumed three-photon allowed, but single-photon forbidden. Examples are afforded by excited states of $A_{2}$ symmetry, in molecules of $C_{2 v}$ or $C_{3 v}$ symmetry, or states of $A_{u}$ symmetry in $D_{2 h}$ species. In such a case, switching action is enabled since the throughput or absence of the laser input will cause activation or deactivation of the fluorescence, respectively. Clearly it is necessary for the radiation to be 
delivered in a pulse whose duration and delay, both with respect to the initial excitation, are sufficiently short that it can engage with the system before there is significant non-radiative dissipation of the excited state.

The result for this case is secured on completion of a rotational-average requiring the rarely utilized eighth-rank isotropic tensor average, ${ }^{5,59}$ requiring manipulations with a $105 \times 105$ matrix. The calculation leads to the following result:

$$
\begin{aligned}
\left\langle I^{\prime}\left(\Omega^{\prime}\right)\right\rangle & =\left(\frac{I^{2}}{84 c^{2} \varepsilon_{0}^{2}}\right) \Xi\left(I_{0}, \omega_{0}\right)\left[3 T_{i(i j j)} \bar{T}_{k(k l l)}\left(3 \cos ^{2} \theta \cos ^{2} \varphi-\cos ^{2} \theta-\cos ^{2} \varphi+\cos ^{2} \phi\right)\right. \\
& +6 T_{i(i j k)} \bar{T}_{j(k l l)}\left(6 \cos \theta \cos \varphi \cos \phi-\cos ^{2} \theta-2 \cos ^{2} \varphi-2 \cos ^{2} \phi+1\right) \\
& +3 T_{i(i j k)} \bar{T}_{l(j k l)}\left(\cos ^{2} \theta \cos ^{2} \varphi-4 \cos \theta \cos \varphi \cos \phi+5 \cos ^{2} \theta+\cos ^{2} \varphi+4 \cos ^{2} \phi-3\right) \\
& +3 T_{i(j j k)} \bar{T}_{i(k l l)}\left(-\cos ^{2} \theta \cos ^{2} \varphi-4 \cos \theta \cos \varphi \cos \phi+\cos ^{2} \theta+5 \cos ^{2} \varphi+\cos ^{2} \phi-1\right) \\
& \left.+T_{i(j k l)} \bar{T}_{i(j k l)}\left(-\cos ^{2} \theta \cos ^{2} \varphi+4 \cos \theta \cos \varphi \cos \phi-5 \cos ^{2} \theta+\cos ^{2} \varphi-4 \cos ^{2} \phi+7\right)\right] .
\end{aligned}
$$

Here, the $\mathbf{T}$ tensors accommodate sums over products of transition moments that specifically exclude $\mu^{0 \alpha}$, on the basis of the decay transition being symmetry-forbidden under electric dipole selection rules;

however, for simplicity, we retain the assumption of Kleinman index symmetry in the embedded $\chi$ tensor (corresponding to the last three indices in each $\mathbf{T}$ ). We observe that the emission depends primarily on presence of the probe laser light, providing a basis for optically switchable emission.

\subsection{Complete result for a two-level system}

For completeness, although the above result must apply to emission from an indirectly excited state, one can adopt the corresponding result for a case of electric dipole-allowed emission and thereby provide a 
completely general result for the probe-modified fluorescence anisotropy, accommodating all of the terms arising from equation (1). Taking once again the two-level model for the emission, we have:

$$
r^{\prime}=\frac{3 \cos ^{2} \beta-1+K I\left|\mu^{0 \alpha}\right|^{2}\left(\cos ^{2} \beta-1\right)+\left(K^{2} I^{2}\left|\mu^{0 \alpha}\right|^{4} / 21\right)\left(15 \cos ^{2} \beta-17\right)}{5+\left(K I\left|\boldsymbol{\mu}^{0 \alpha}\right|^{2} / 7\right)\left(20-11 \cos ^{2} \beta\right)+\left(K^{2} I^{2}\left|\boldsymbol{\mu}^{0 \alpha}\right|^{4} / 21\right)\left(43-30 \cos ^{2} \beta\right)} .
$$

In a case where the absorption and transition moments are parallel, we secure the simple result:

$$
r^{\prime}=\frac{2-2\left(K^{2} I^{2}\left|\boldsymbol{\mu}^{0 \alpha}\right|^{4} / 21\right)}{5+9\left(K I\left|\boldsymbol{\mu}^{0 \alpha}\right|^{2} / 7\right)+13\left(K^{2} I^{2}\left|\boldsymbol{\mu}^{0 \alpha}\right|^{4} / 21\right)}
$$

With increasing intensity of the probe beam, the first departures from the probe-free result, $r^{\prime}=0.4$, can be anticipated in the linear-response regime. In fact, it is evident from Taylor series expansions of both (13) and (14) that a plot of the measured anisotropy against $I$ will exhibit a monotonic reduction taking

the form $r^{\prime} \approx \frac{1}{5}\left(3 \cos ^{2} \beta-1\right)(1-\eta I)$, whose constant of proportionality $\eta$ can be interpreted in terms of the transition moments.

\section{Conclusion}

Building on earlier work, the theory of laser-controlled molecular fluorescence has been developed in order to elicit a number of features of particular experimental significance. Use of the two-level emission model is widely valid for systems including those that incorporate common fluorescent markers, and here it proves to offer succinct and experimentally tractable results. The analysis has thus 
been shown to deliver results of broad applicability - whose simplified form, without further approximation, is consistent with the adoption of Kleinman symmetry. Equations have been derived for

the anisotropy of fluorescence that can be expected from a system responding to the passage of offresonant light, its leading correction being linearly dependent on the probe irradiance and manifest as a reduction of the measured anisotropy. It has also been shown that, for some electronic states which normally decay non-radiatively, it is possible to optically switch fluorescent emission using the offresonance probe. In all such respects, the capacity to engage with and to optically control the fluorescence process offers significant new grounds for the interrogation of fluorescent materials.

\section{Acknowledgements}

The authors are grateful to EPSRC for financial support.

\section{References}

1. Marsh, R. J.; Leonczek, N. D.; Armoogum, D. A.; Porres, L.; Mongin, O.; Blanchard-Desce, M.; Bain, A. J. Proc. SPIE 2004, 5510, 117.

2. Kastrup, L.; Blom, H.; Eggeling, C.; Hell, S. W. Phys. Rev. Lett. 2005, 94, 178104.

3. Hell, S. W. Science 2007, 316, 1153.

4. Willig, K. I.; Harke, B.; Medda, R.; Hell, S. W. Nature Methods 2007, 4, 915.

5. Zhou, L. C.; Liu, J. Y.; Zhao, G. J.; Shi, Y.; Peng, X. J.; Han, K. L. Chem. Phys. 2007, 333, 179.

6. Armoogum, D. A.; Marsh, R. J.; Nicolaou, N.; Mongin, O.; Blanchard-Desce, M.; Bain, A. J. Proc SPIE 2008, 7030, 70300S. 
7. Harke, B.; Keller, J.; Ullal, C. K.; Westphal, V.; Schoenle, A.; Hell, S. W. Opt. Exp. 2008, 16, 4154.

8. Bradshaw, D. S.; Andrews, D. L. J. Phys. Chem. A 2009, 113, 6537.

9. Bradshaw, D. S.; Andrews, D. L. Phys. Rev. A 2010, 81, 013424.

10. Andrews, D. L.; Leeder, J. M.; Bradshaw, D. S. Proc. SPIE 2010, 7712, 77121R.

11. Andrews, D. L.; Bradshaw, D. S. Opt. Commun. 2010, 283, 4365.

12. Rigneault, H.; Capoulade, J.; Dintinger, J.; Wenger, J.; Bonod, N.; Popov, E.; Ebbesen, T. W.; Lenne, P.-F. Phys. Rev. Lett. 2005, 95, 117401.

13. Anger, P.; Bharadwaj, P.; Novotny, L. Phys. Rev. Lett. 2006, 96, 113002.

14. Yu, Y.-J.; Noh, H.; Jhe, W.; Noh, H.-R.; Nakaoka, T.; Arakawa, Y. Phys. Rev. B 2010, 82, 085308 .

15. Spano, F. C.; Mukamel, S. Phys. Rev. A 1989, 40, 5783.

16. Leegwater, J. A.; Mukamel, S. Phys. Rev. A 1992, 46, 452.

17. Jenkins, J. K.; Mukamel, S. J. Chem. Phys. 1993, 98, 7046.

18. Mukamel, S. Principles of Nonlinear Optical Spectroscopy; Oxford University Press: New York, 1995.

19. Chernyak, V.; Tretiak, S.; Mukamel, S. Chem. Phys. Lett. 2000, 319, 261.

20. Venkatramani, R.; Mukamel, S. J. Phys. Chem. B 2005, 109, 8132.

21. Rahav, S.; Mukamel, S. Proc. Natl. Acad. Sci. 2010, 107, 4825.

22. Zrenner, A.; Beham, E.; Stufler, S.; Findeis, F.; Bichler, M.; Abstreiter, G. Nature 2002, 418, 612.

23. Klimov, V. I. Los Alamos Science 2003, 28, 214.

24. Kirkpatrick, S. M.; Naik, R. R.; Stone, M. O. J. Phys. Chem. B 2001, 105, 2867.

25. Drobizhev, M.; Marakov, N. S.; Hughes, T.; Rebane, A. J. Phys. Chem. B 2007, 111, 14051.

26. Asselberghs, I.; Flors, C.; Ferrighi, L.; Botek, E.; Champagne, B.; Mizuno, H.; Ando, R.; Miyawaki, A.; Hofkens, J.; van der Auweraer, M.; Clays, K. J. Am. Chem. Soc. 2008, 130, 15713. 
27. Drobizhev, M.; Tillo, S.; Makarov, N. S.; Hughes, T. E.; Rebane, A. J. Phys. Chem. B 2009, 113, 12860.

28. Beuerman, E.; Makarov, N. S.; Drobizhev, M. Proc. SPIE 2010, 7599, 75990X.

29. Allcock, P.; Jenkins, R. D.; Andrews, D. L. Phys. Rev. A 2000, 61, 023812.

30. Andrews, D. L.; Crisp, R. G. Proc. SPIE 2005, 5924, 167.

31. Andrews, D. L.; Crisp, R. G. J. Opt. A: Pure Appl. Opt. 2006, 8, S106.

32. Andrews, D. L. Phys. Rev. Lett. 2007, 99, 023601.

33. Andrews, D. L.; Crisp, R. G.; Li, S. J. Chem. Phys. 2007, 127, 174702.

34. Bradshaw, D. S.; Andrews, D. L. J. Chem. Phys. 2008, 128, 144506.

35. Bradshaw, D. S.; Andrews, D. L. Appl. Phys. B 2008, 93, 13.

36. Bradshaw, D. S.; Andrews, D. L. Laser Phys. 2009, 19, 125.

37. Bradshaw, D. S.; Andrews, D. L. J. Nanophotonics 2009, 3, 031503.

38. Bradshaw, D. S.; Andrews, D. L. Superlatt. Microstruct. 2010, 47, 308.

39. Mandel, L.; Wolf, E. Optical Coherence and Quantum Optics; University Press: Cambridge, U.K., 1995 , p. 871.

40. Craig, D. P.; Thirunamachandran, T. Molecular Quantum Electrodynamics; Dover: Mineola, N.Y., 1998.

41. Andrews, D. L.; Allcock, P. Optical Harmonics in Molecular Systems; Wiley-VCH: Weinheim, Germany, 2002.

42. Kleinman, D. A. Phys. Rev. 1962, 126, 1977.

43. Oudar, J. L.; Chemla, D. S. J. Chem. Phys. 1977, 66, 2664.

44. Leasure, S. C.; Wyatt, R. E. Opt. Eng. 1980, 19, 46.

45. Dick, B.; Hohlneicher, G. J. Chem. Phys. 1982, 76, 5755.

46. Meath, W. J.; Power, E. A. J. Phys. B: At. Mol. Opt. Phys. 1984, 17, 763.

47. Meath, W. J.; Power, E. A. Mol. Phys. 1984, 51, 585. 
48. Kmetic, M. A.; Meath, W. J. Phys. Lett. A 1985, 108, 340.

49. Wortmann, R.; Krämer, P.; Glania, C.; Lebus, S.; Detzer, N. Chem. Phys. 1993, 173, 99.

50. Kondo, A. E.; Meath, W. J.; Nilar, S. H.; Thakkar, A. Chem. Phys. 1994, 186, 375.

51. Tran, P.; Meath, W. J.; Wagner, B. D.; Steer, R. P. J. Chem. Phys. 1994, 100, 4165.

52. Jagatap, B. N.; Meath, W. J. Chem. Phys. Lett. 1996, 258, 293.

53. Andrews, D. L.; Dávila Romero, L. C.; Meath, W. J. J. Phys. B: At. Mol. Opt. Phys. 1999, $32,1$.

54. Dávila Romero, L. C.; Andrews, D. L. J. Phys. B: At. Mol. Opt. Phys. 1999, 32, 2277.

55. Andrews, D. L.; Dávila Romero, L. C. J. Phys. B: At. Mol. Opt. Phys. 2001, 34, 2177.

56. Juzeliūnas, G.; Dávila Romero, L. C.; Andrews, D. L. Phys. Rev. A 2003, 68, 043811.

57. Valeur, B. Molecular Fluorescence; Wiley-VCH: Weinheim, 2002.

58. Andrews, D. L.; Ghoul, W. A. J. Phys. A: Math. Gen. 1981, 14, 1281.

59. Andrews, D. L.; Ghoul, W. A. J. Chem. Phys. 1981, 75, 530. 


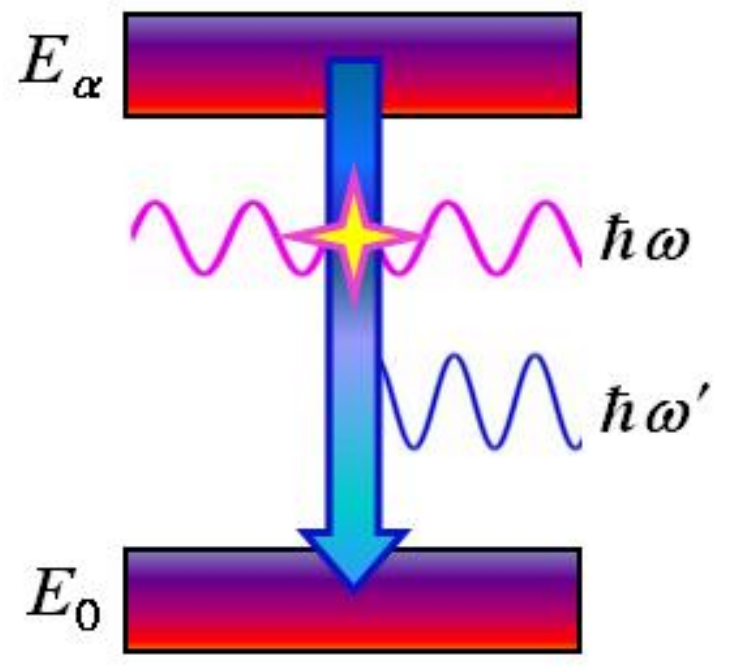

TOC figure: Artist's impression of laser-controlled fluorescence 